\title{
PROBLEMS OF THE COMPENSATION METHODS FOR THE SPIN DEPOLARIZING RESONANCES IN THE STRONG FOCUSING SYNCHROTRON
}

\author{
Hikaru Sato \\ National Laboratory for High Energy Physics \\ KEK, Tsukuba, Japan
}

May 15, 1990

\section{DISCLAIMER}

This report was prepared as an account of work sponsored by an agency of the United States Government. Neither the United States Gnvernment nor any agency thereof, nor any of their employees, makes any warranty, express or implied, or assumes any legal liability or responsibility for the accuracy, completeness, or usefulness of any information, apparatus, product, or process disclosed, or represents that its use would not infringe privately owned rights. Reference herein to any specific commercial product, process, or service, by trade name, trademark, manufacturer, or otherwise does not necessarily constitute or imply its endorsement, recommendation, or favoring by the United States Government or any agency thereof. The views and apinions of authors expressed herein do not necessarily state or reflect those of the United States Government or any agency thereof.

\section{ALTERNATING GRADIENT SYNCHROTRON DEPARTMENT}

BROOKHAVEN NATIONAL LABORATORY

UPTON, LONG ISILAND, NEW YORK 11973 
Problems of the Compensation Methods for the Spin Depolarizing Resonances in the Strong Focusing Synchrotron

\author{
Hikaru Sato \\ National Laboratory for High Energy Physics \\ 1-1 Oho,Tsukuba-shi,Ibaraki-ken,305 Japan
}

Introduction

The achievement of a high energy polarized proton beam requires the reduction of depolarization during acceleration. This depolarization will occur at spin resonances where the spin precession frequency equals that of a horizontal magnetic field component. There are basically two types of first order depolarizing resonances. One type is an intrinsic resonance which is excited by the periodical focusing structure of the machine. This resonance occurs at $\gamma \mathrm{G}=\mathrm{nN} \pm v_{z}$, where $\gamma, G, n, N$ and $v_{z}$ are the Lorentz energy factor, the gyromagnetic factor, an integer, the superperiodicity number of the machine and the vertical betatron tune, respectively. The resonance strength is proportional to the vertical betatron oscillation amplitude. The other type is an imperfection resonance which is due to the magnet misalignment leading to vertical closed orbit distortion (COD). This resonance occurs at $\gamma \mathrm{G}=\mathrm{nN} \pm \mathrm{k}$, where $\mathrm{k}$ is the harmonic number of the vertical COD. The resonance strength is proportional to the amplitude of the vertical COD.

There can be also higher order resonances caused by higher order nonlinear magnetic fields in the machine and the synchrotron oscillation effect if the depolarizing resonance is passed by an adiabatic spin flip. The research and development to accelerate the polarized beam is being 
performed in Saturne II at Saclay," AGS at Brookhaven National Laboratory ${ }^{2)}$ and the KEK PS. ${ }^{3)}$ Table 1 shows the present status for the polarized proton acceleration of these machines.

Table 1

Machine $\begin{gathered}\text { Energy Polarization } \\ \text { or Momentum }\end{gathered} \begin{gathered}\text { Beam } \\ \text { Intensity }\end{gathered} \begin{gathered}\text { Max.Energy } \\ \text { of machine }\end{gathered}$ Final goal

AGS $\quad 22 \mathrm{GeV} / \mathrm{c} \quad 45 \% \quad 1-2 \times 10^{10} \quad 30 \mathrm{GeV} \quad 26 \mathrm{GeV} / \mathrm{c}, 60 \%$ KEK PS $\quad 3.5 \mathrm{GeV} \quad 40 \% \quad 0.9 \times 10^{10} 12 \mathrm{GeV} \quad 12 \mathrm{GeV}, 40 \%$ Saturne II $2.9 \mathrm{GeV} \quad 80 \% \quad 2 \times 10^{11} 2.95 \mathrm{GeV}$

The theory and technique seemed to be well established up to a few teris of $\mathrm{GeV}$, however there are several problems to achieve the final goal, though several experiments have been run from 1984 at the AGS ${ }^{4)}$ and 1986 at the KEK PS, ${ }^{5,6)}$ respectively. This paper reports on phenomena and problems about depolarizing resonances encountered in accelerating polarized protons at the AGS and the KEK PS.

\section{A Brief Review of Polarized Proton Beam Acceleration}

During acceleration of the polarized beam in a synchrotron, many depolarizing resonances must be passed through as the beam energy increases. The polarization $P_{f}$ after passing through each isolated depolarizing resonance is given by, ?

$$
P_{f}=P_{i}\left(2 e^{-\pi \varepsilon^{2} / 2 \alpha}-1\right)
$$


where $P_{i}$ is the polarization before passing through the resonance, $\varepsilon$ is the resonance strength, $\alpha$ is the rate of passage,

$$
\alpha=\left(\dot{\gamma} \mathrm{G} \pm \dot{v}_{z}\right) / \omega_{0}
$$

for an intrinsic resonance, and

$$
\alpha=\dot{\gamma} \mathrm{G} / \omega_{0}
$$

for an imperfection resonance, where $\omega_{0}$ is the angular frequency of the beam revolution. There are several methods to reduce the depolarization expected from equation ( 1 ).

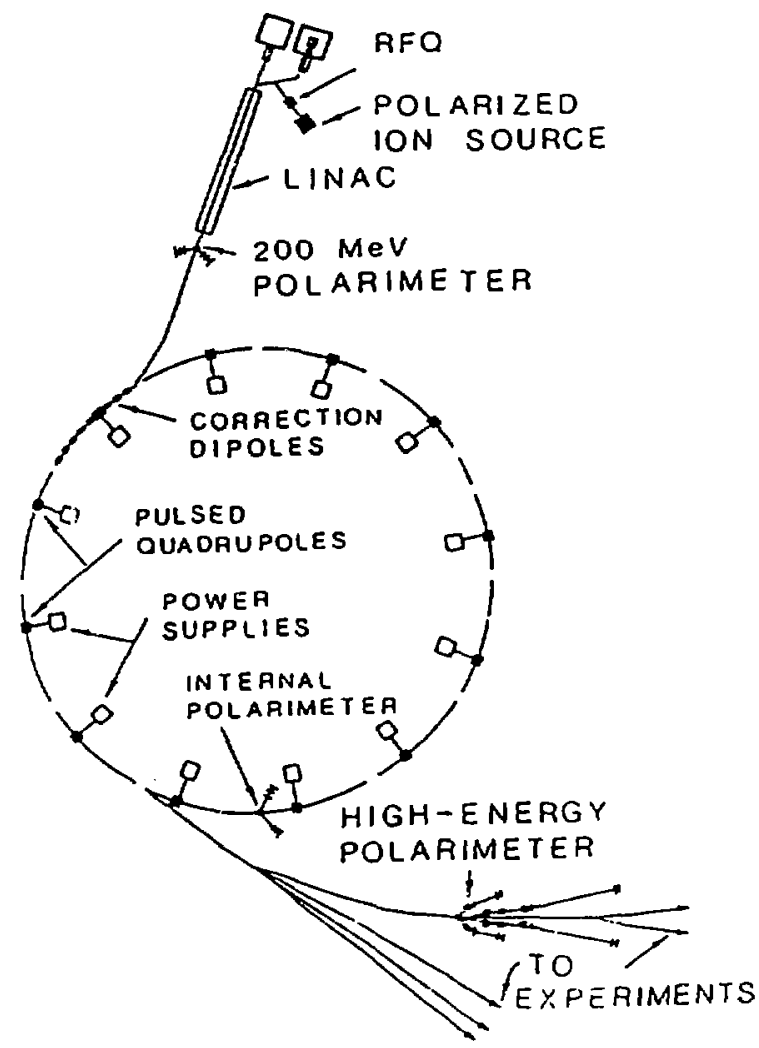

Fig.1. AGS layout for the operation of the polarized proton beam. 
If $\varepsilon^{2} / \alpha$ is small enough then the polarization is preserved, on the other hand, if $\varepsilon^{2} / \alpha$ is sufficiently large the spin will flip with no depolarization.

Table 2 and 3 show the depolarizing resonance strengths at the AGS ${ }^{8)}$ and KEK PS 9).10), respectively. Here, the author defines the intrinsic resonance width to be $7 \varepsilon$ to require $99 \%$ preservation of polarization. ${ }^{11) *}$ Generally, pulsed quadrupole magnets are used to increase the crussing speed through the intrinsic resonance and correction dipoles are used for the harmonic correction of the vertical COD. Figures 1 and 2 show the equipment for the polarized proton beam acceleration at thie AGS and the KEK PS, respectively.

$750 \mathrm{keV}$

preaccelerator

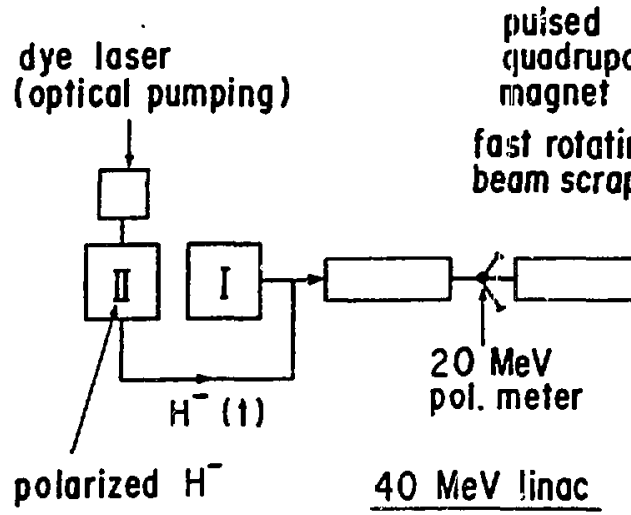

ion source mognet

\section{$500 \mathrm{MeV}$ booster $\quad 12 \mathrm{GeV}$ moin ring}

puised pulsed dipole quadrupole mognet

(orbit corr.)

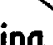

.

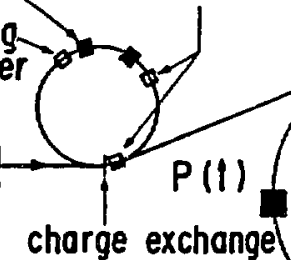

injection

$\left(\begin{array}{l}\text { C- foil } \\ \sim 30 \mu \mathrm{g} / \mathrm{cm}^{2}\end{array}\right)$
$500 \mathrm{MeV} \quad$ moin ring

pol. meter pol.meter 
Table 2 (AGS)

$$
\begin{aligned}
& N=12 \\
& v_{z}=8.75 \\
& \alpha=4.86 \times 10^{-5} \\
& d(\gamma G) / d t=95 / \mathrm{sec}
\end{aligned}
$$

Table 3 (KEK PS)

$$
\begin{aligned}
& N=4 \\
& v_{z}=6.25 \\
& \alpha=5.8 \times 10^{-6} \\
& d(\gamma G) / d t=25 / \mathrm{sec}
\end{aligned}
$$

Intrinsic resonances

Fast jumping method.

It is possible to increase $\alpha$ by rapidly changing the vertical betatron tune $v_{z}$ when $\gamma G$ approaches the resonance in order to reduce the depolarization. Figures 3 and 4 show some typical correction scheme and correction curve by fast tune jump, respectively. This method has been used quite successfully at the AGS ${ }^{12)}$ and the KEK PS ${ }^{9) .10)}$, however, there are some problems in this method. The jumping width should be decided by not only the resonance width but also the momentum spread and the tune spread caused by the chromaticity. ${ }^{11}$ ) The jumping width has 


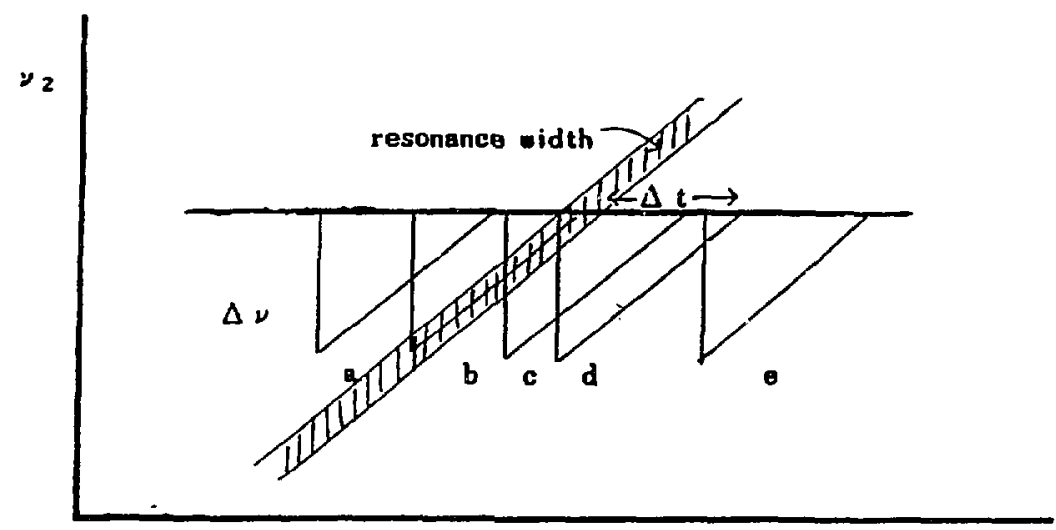

Firing Time of Fast Quad

Fig.3. Illustration of the fast passage method.

Periods a,b,c,d and e correspond to those in Fig.4.

$t$ is the time margin for fast jump.

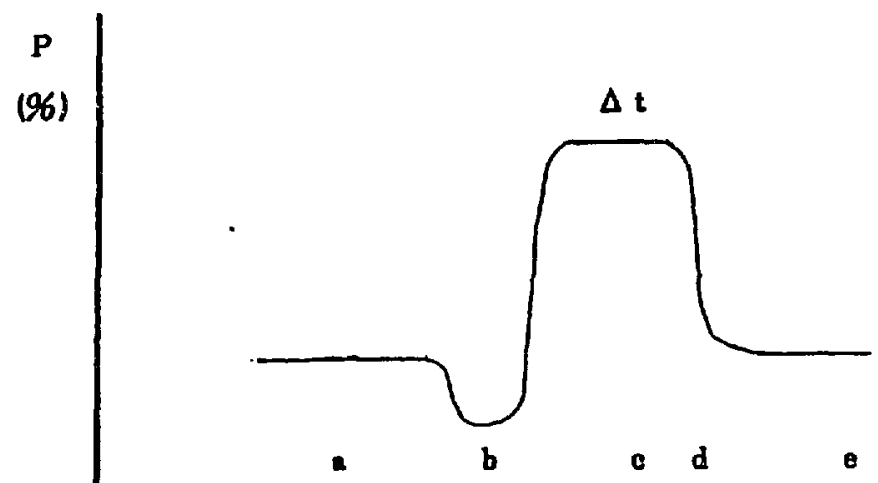

Fring Time of Fast Quad

Fig.4. Typical correction curve for the fast passage method.

Periods $a, b, c, d$ and e correspond to those in Fig.3.

$t$ is time margin for fast jump. 
to be limited to avoid hitting integer or half-integer resonance. Since the nominal tune is near an integer \pm 0.25 ( such as 8.75 at AGS and 6.25 at KEK PS ), if a larger than 0.25 tune shift is required, the tune jump would hit either an integer or half-integer resonance. In order to avoid this, a slow tune shift would be required as shown in Fig. 5. Even if the slow tune shift would be adopted, it is difficult for the jumping width to be more than 0.3. Then, this method is not available for the strong intrinsic resonance whose strength is larger than $\varepsilon=0.02$. The narrow plateau as shown in Fig. $6^{12)}$ indicates this effect for the resonance, $\gamma \mathrm{G}=\mathrm{V}_{z}$ at the AGS.

The fast pulsed quadrupoles in the AGS are ferrite magnets with a $2 \mu \mathrm{sec}$ rise time to cross each resonance in less than one beam revolution. ${ }^{4) .13)}$ On the other hand, the rise time of fast quadrupoles in the KEK PS is $40 \mu \mathrm{sec}$ to $200 \mu \mathrm{sec}$ which was designed close to the critical speed in considering the calculated resonance strength to save magnet and pulsed power supply costs. ${ }^{11)}$ The results which were obtained in the run up to $5.0 \mathrm{GeV}^{\text {9) }}$ as shown in Fig. 7 indicate that these pulsed magnets are sufficiently good for fast crossing, however, it is not certain for the higher energy region. S.Y.Lee pointed our that the tune jump method becomes impractical at larger resonance strength than 0.05 even if the tune jump was performed within one revolution. ${ }^{14)}$

From the view point of beam orbit dynamics, the betatron amplitude function is increased by the fast quadrupole magnet excitation ,especially for an asymmetric configuration. The deformation of the beta function becomes large when the tune value is close to a half integer. ${ }^{(3)}$ Funhermore, misalignment of the fast quadrupole causes closed orbit errors. At the AGS the emittance growth caused by the fast quadrupole magnets was observed in the $1986 \mathrm{run}$, and it was greatly reduced by the realignment of the AGS in the 1988 run as shown in Fig. ${ }^{21.14}$ ). 16) The 
increase of the beam emittance is translated into an increase of the intrinsic resonance strength, so careful alignment of the machine is required for polarized beam acceieration.

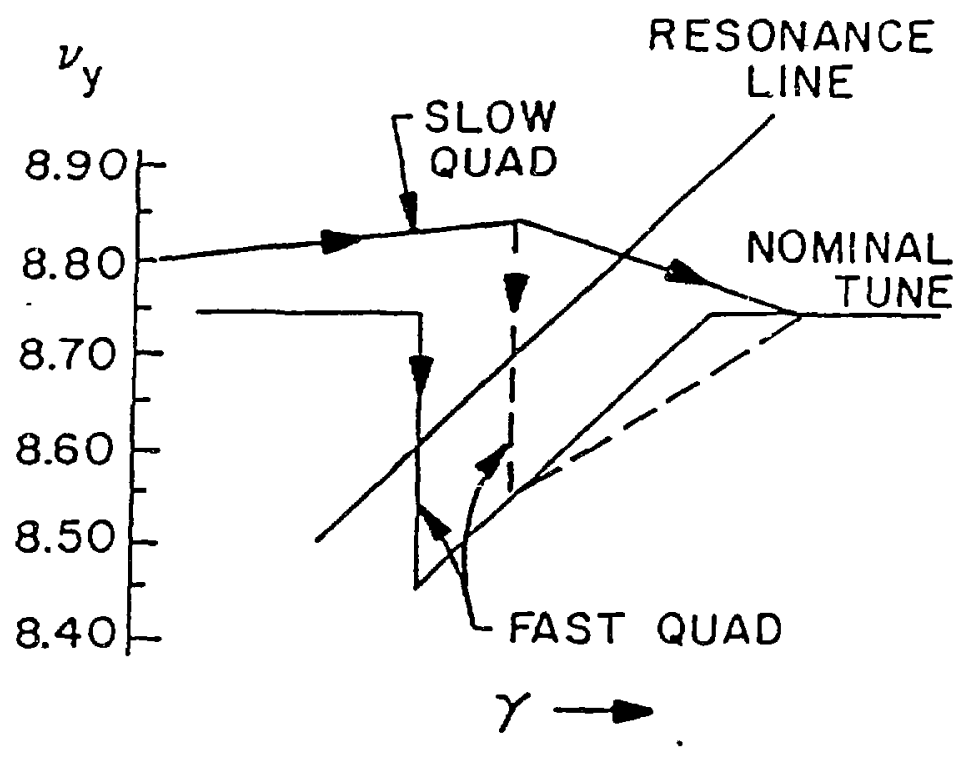

Fig.5. Slow tune shifting for large amount fast jump.

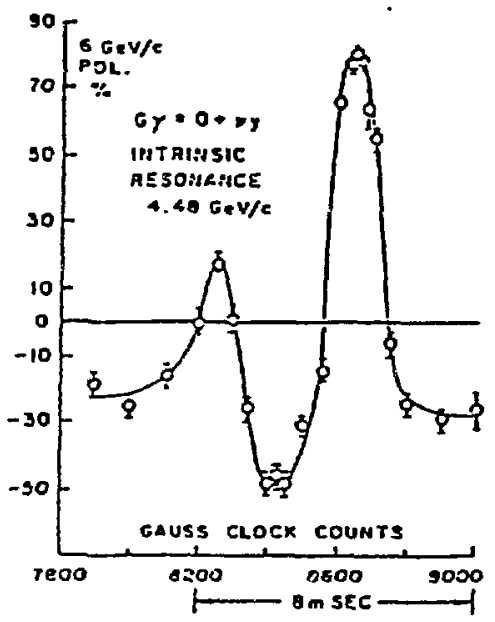

Fig.6. Correction curve for the $\gamma \mathrm{G}=\mathrm{v}_{2}$ intrinsic resonance at the AGS. 


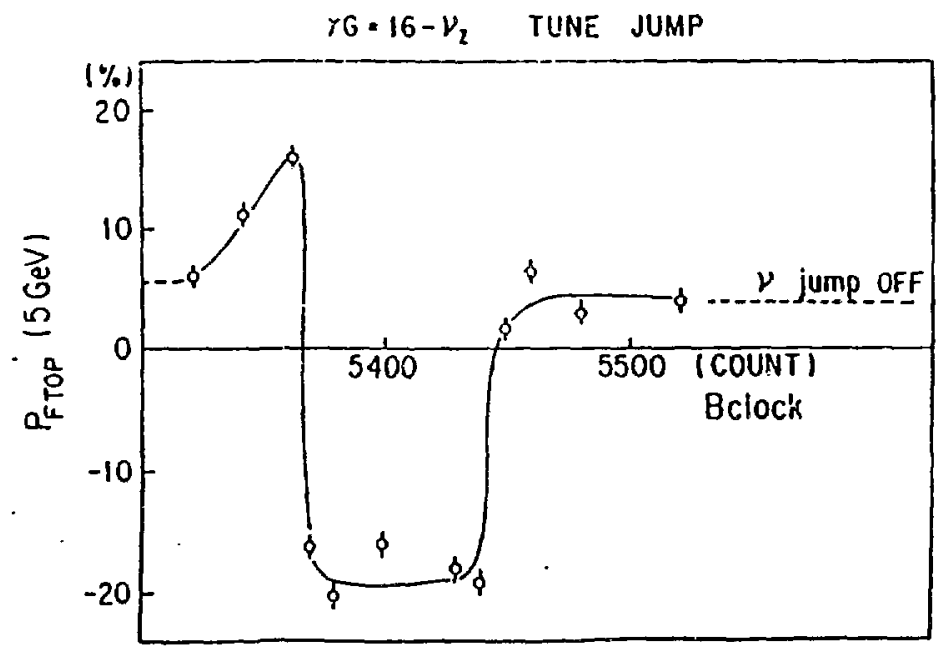

Fig.7. Correction curve for the $\gamma G=16-v_{2}$ intrinsic resonance at the KEK PS main ring.

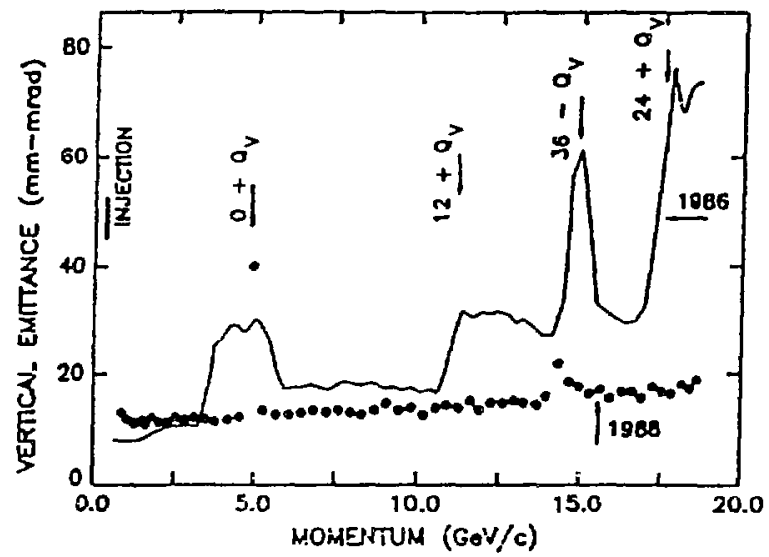

Fig.8. Emittance growth caused by the fast quadrupole magnet at the AGS. 
Spin flip

It is possible to pass the depolarizing resonance with an adiabatic spin flip by decreasing the crossing speed, $\alpha$, or increasing the vertical beam size to flip the spin. However, there are several limitations or problems in this method. The particles which are distributed in the vertical phase space have various resonance strengths depending on each betatron oscillation amplitude, and the spin flipped beam polarization has to be averaged over in the vertical phase space. A calculation with an assumed distribution, for example, uniform or quadrutic distribution, in verical phase space shows that the idealized maximum beam polarization would be $90-95 \%$.

Increasing the vertical beam size is limited by the aperture and the problem of increasing the resonance strength of the next intrinsic resonances. Decreasing $\alpha$ by adjusting the falling tail of the pulsed quadrupole magnetic field is a rather attractive method, bit it has the same problem of the fast jump, that is, $\Delta v$ must not be more than 0.3 . If the accelerating speed is much faster, then it is difficult to pass the strong resonances at une AGS by spin flip. Although the results are insufficient, these effects are confirmed at the $\gamma \mathrm{G}=4+v_{z}$ resonance in the KEK PS, ${ }^{\text {) }}$ as shown in Fig. 9, where the accelerating speed is slower than that of the AGS.

Furthermore, there is a severe problem in this method. It is the depolarizing effect caused by the synchrotron oscillation. This phenomena was first observed at the $\gamma \mathrm{G}=2$ imperfection resonance in Saturne II. ${ }^{17)}$ The beam is a collection of particles bunched by the accelerating if as well as confined in the transverse space by a focusing field. Each particle oscillates in energy around the synchronous energy at the synchrotron oscillation frequency. This oscillation causes not only a modulation of the spin precession frequency in one revolution of the 


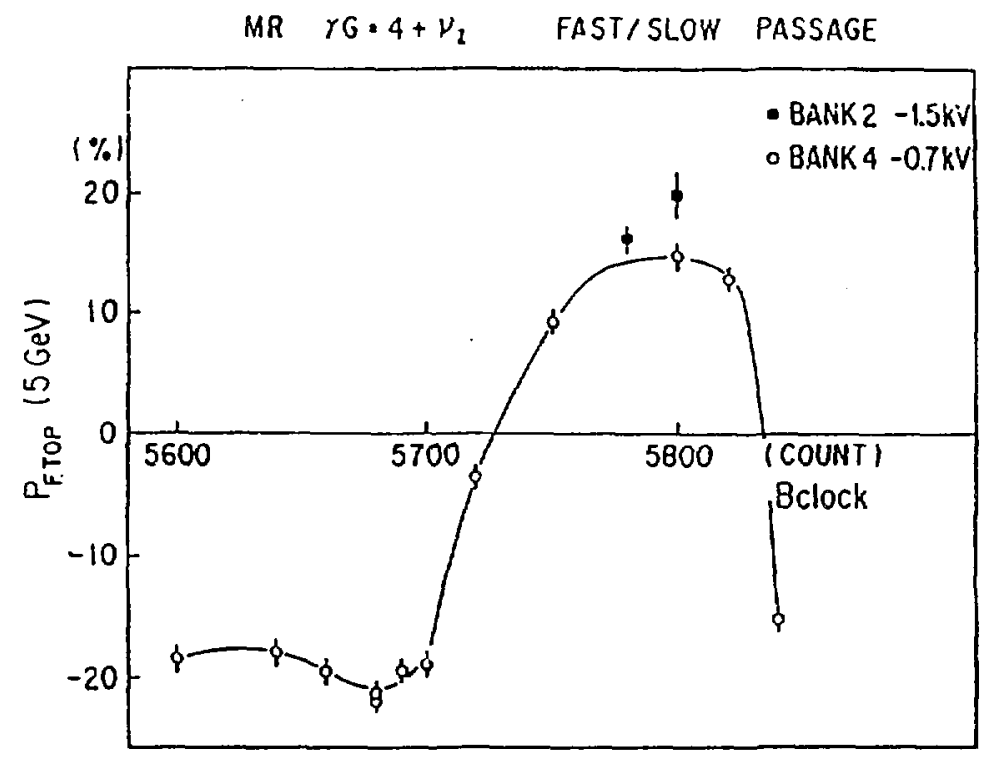

Fig.9. Correction curve for the $\gamma \mathrm{G}=4+v_{2}$ intrinsic resonance at the KEK PS main ring.

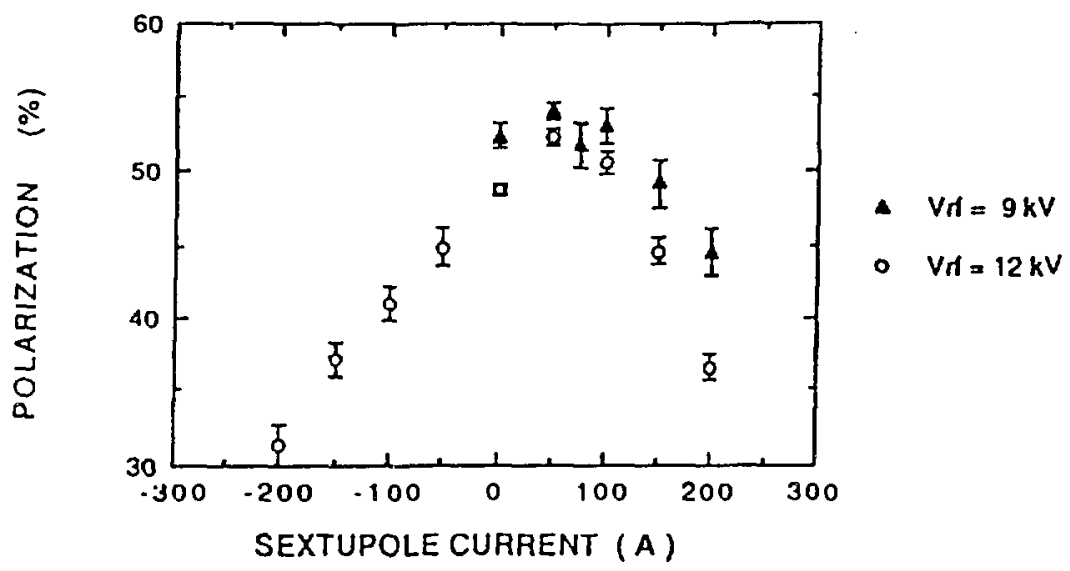

Fig.10. Sextupole current dependence of the $\gamma G=v_{z}$ intrinsic resonance at the KEK PS booster. 
particle but also a modulation of the resonant frequency, $\mathrm{nN} \pm v_{z^{*}}$. This modulation will lead to multiple resonance crossing and consequently cause depolarization. This frequency is proportional to the square root of the if voltage. Therefore, reducing the $f f$ voltage can be used to eliminate the multiple resonance crossing, ${ }^{3)}$ but this is limited by the longitudinal acceptance.

A more attractive method is adjusting the vertical chromaticity to satisfy the condition of $\xi_{z}= \pm \gamma_{0} G \beta^{2}{ }^{18)}$.19) This method was confimed in a KEK PS polarized proton beam acceleration study as shown in Fig.10. However, more detailed consideration and study will be necessary to accelerate the beam stably. ${ }^{19)}$ Tizere is one weakness of this method. The value of correction chromaticity is proportional to $\gamma$, so it is availatle only for the low energy region. Further, the beam distribution in the vertical phase space must be taken into consideration in order to investigate the final beam polarization after crossing several strong intrinsic resonances by the spin flip.

\section{Imperfection resonance}

The strength of the imperfection resonance is proportional to the harmonic component amplitude of the vertical COD. Therefore the resonance strength can be controlled by the harmonic component tuning of the vertical COD. The orbit correction can be done by using a set of vertical steering dipole magnets which correct the error field in the ring magnets.

Although the harmonic correction method is effective to correct the imperfection resonances, there are several problems. The excitation current of the correction dipoles is proportional to the energy of the particle. Further there is not only one harmonic component for the 
higher energy as identified from the equation $\gamma \mathrm{G}=\mathrm{nN} \pm \mathrm{k}$, so rather complicated correction tuning must be performed.9) Ninety six and twenty eight correction dipoles were used at the AGS and the KEK PS, respectively. The imperfection and intrinsic beat resonance will occur when the driving imperfection frequency is near the vertical betatron frequency. This resonance should be strong as shown at the $\gamma \mathrm{G}=27$ resonance in the AGS and was reduced by the harmonic correction close to the vertical betatron oscillation. Since $v_{z}=8.75$ and $n N=j 6$ (when $\mathrm{n}=3$ ) for the AGS , 27=36-9, then the 9th harmonic correction reduced the depolarization successfulıy instead of direct correction of the 27th harmonic component as shown in Fig.11. 4).20)

Imperfection resonances which are due to the magnet misalignments would change with an alignment of the ring magnets. The amplitude and the phase of COD depend on the tune value, for example, the 6th harmonic of vertical COD of KEK PS is shown in Fig.12 against the vertical tune.

When slow tune shifting is used to make a large fast tune change, for the strong intrinsic resonance, it can translate into the variation of the vertical COD. Then, it must be necessary to perform complicated tuning of the jump timing and harmonic correction.

If there is a margin in the dynamic beam aperture, the method of spin flip by overcorrection of the COD is an easy tuning method to reduce the depolarization. The operating point can be selected when there is an effect of the synchrotron oscillation. This method is used successfully at SATUNE-II ${ }^{21)}$ and KEK PS.9),10) In the 1988 run at the AGS, the accelerating rate was reduced to $60 \%$ of the normal rate due to problems with the main ring power supply, this method was used for several imperfection resonances. ${ }^{2)}$ 

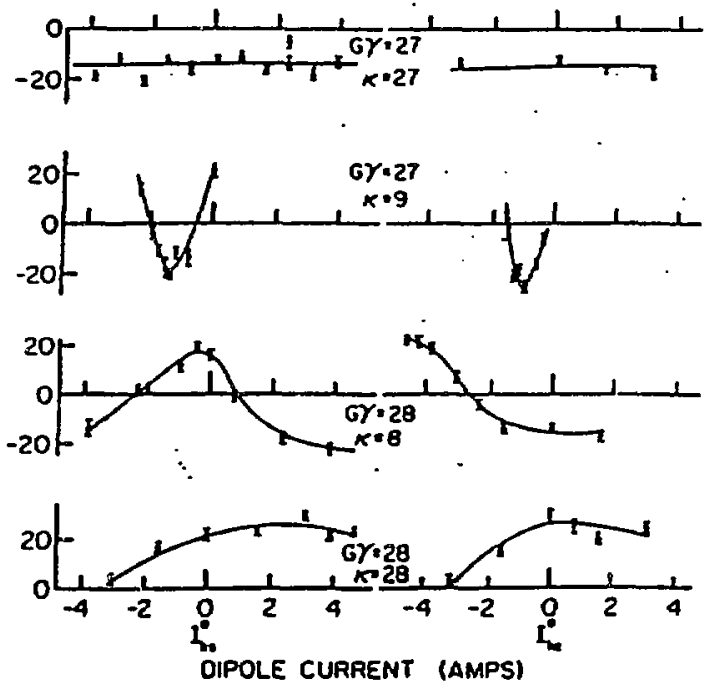

Fig.11. Polarization response to the vertical COD harmonics at the AGS.

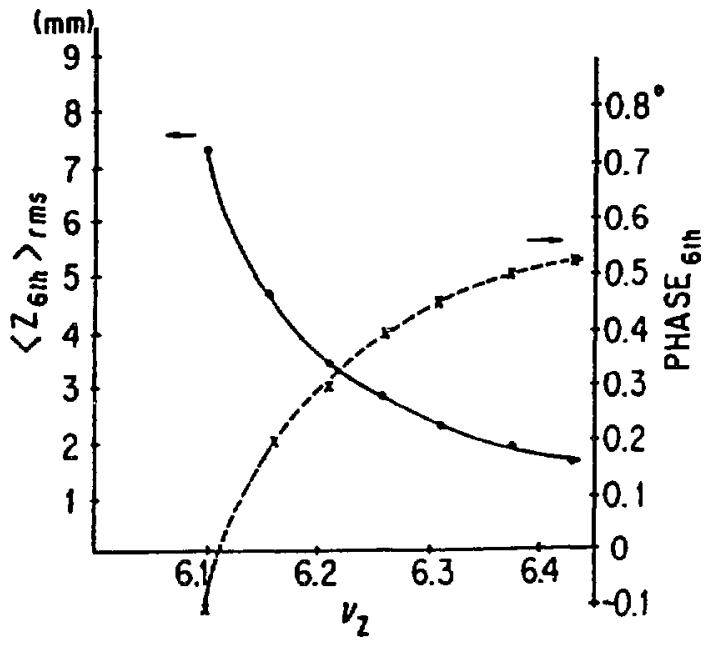

Fig.12. Sixth harmonic of the vertical COD dependence on $v_{z}$ at the KEK PS main ring. 


\section{Higher order resonances}

The higher order resonances can be caused by the higher order nonliriear magnetic fields in the machine and their strengths are generally rather weak. When the extraction energy is in the vicinity of such higher order resonance and beam would be extracted on a long flat top, significant depolarization will be obtained. ${ }^{17,21) 222)}$ So, this flat top field must be adjusted in order to not sit on higher order resonances. If this resonance appears during extraction, it can be avoided by the fast jumping method or controlling the COD in a similar manner to the first order resonance. Resonances due to the horizontal vertical betatron oscillation coupling would be reduced by control of the skew quadrupole magnet component. ${ }^{23)}$

\section{Resonance cluster}

In the 1986 run at the AGS, there is clearly a sharp polarization loss near $14 \mathrm{GeV} .{ }^{24)}$ This problem might be considered to be some sort of interference between the $\gamma \mathrm{G}=36-v_{z}$ intrinsic resonance and the $\gamma \mathrm{G}=27$ imperfection resonance, and it was partly overcome by separating these two resonances by using the slow correction quadrupoles, which shift the vertical tune from 8.75 to 8.55 . (At that time, some depolarization still remained but this problem was not apparent in the 1988 run described above.)

At the KEK PS, a vertical tune of 6.25 is used for the usual polarized proton acceleration. There is a resonance cluster in this condition around $\gamma \mathrm{G}=4 \mathrm{n}+6$ (n=integer), for example, $\gamma \mathrm{G}=12-v_{z}=5.75, \gamma \mathrm{G}=6$ and $\gamma \mathrm{G}=\mathrm{v}_{2}=6.25$ as shown in Fig. 13. When these resonances are strong, the compensation technique will be tricky because they are interfering with 
each other. In order to avoid the resonance cluster, it is better to choose an operating point which does not make a cluster. Generally when $v_{2}=m N / 2 \pm 0.25$ ( $m$ is an integer and $N$ is the superperjodicity) the resonance cluster will appear. Therefore, there is no resonance cluster in the AGS $\left(v_{z}=8.75, N=12\right)$. Although one more intrinsic resonance $\gamma G=$ $-4+v_{z}$ will occur, there is no such resonance cluster in KEK PS when a betatron tune of 7.25 is used. The KEK PS can be operated with both $v_{x}=$ 6.25 and 7.25 , so the study of polarized proton beam acceleration at $v_{z}=$ 7.25 is very desirable.

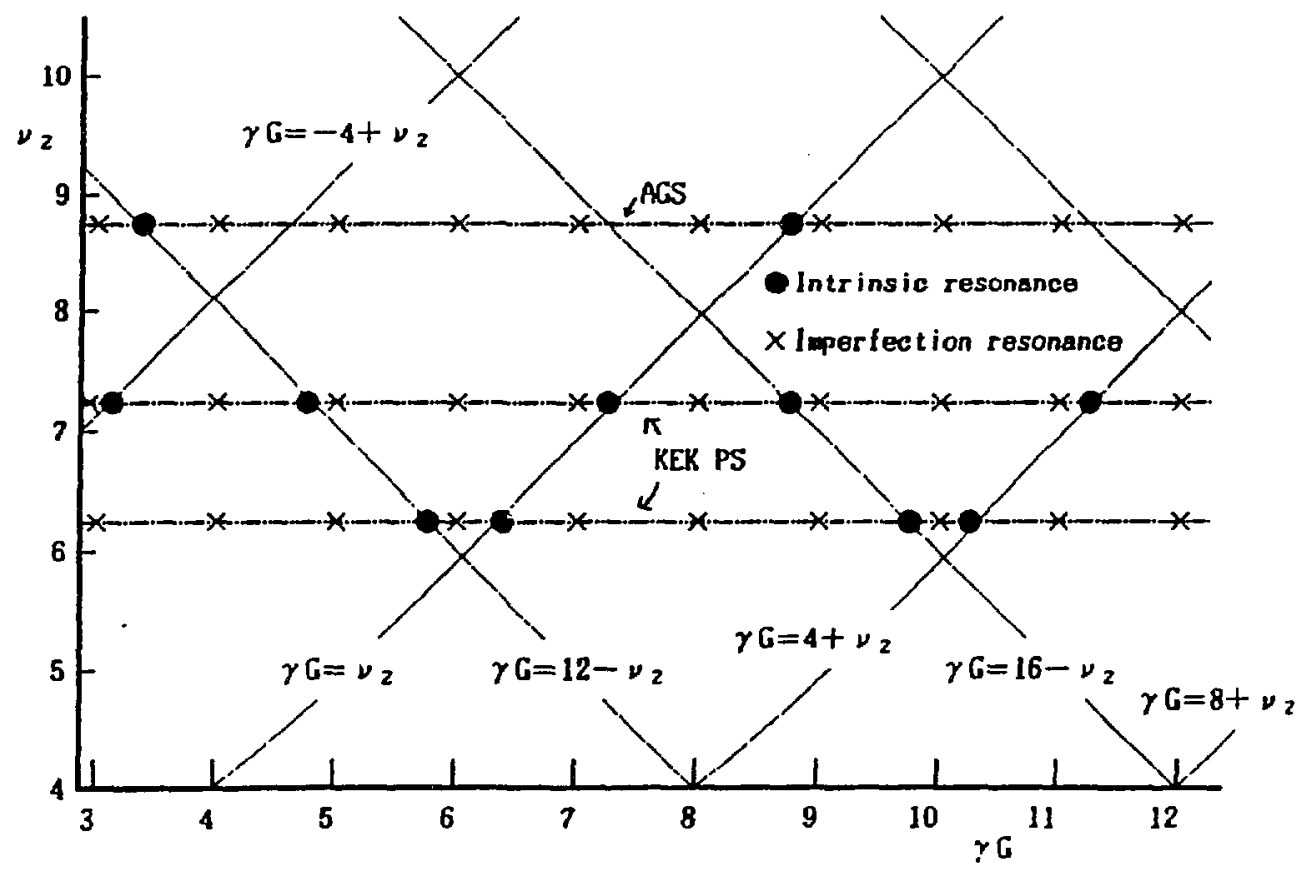

Fig.13. Resonance map up to the $\mathcal{G}=12$ in the tune energy space at the AGS and the KEK PS main ring. 


\section{Internal Polarimeter}

In order to perform experiments using the polarized beam and to assist the polarized beam acceleration development, an external polarimeter is an important tool. For the AGS experiment on spin effects, the high energy polarimeter which contains a liquid hydrogen target measures the proton-proton elastic scattering left-right asymmetry events. ${ }^{4)}$ At the KEK PS, the external polarimeter consists of a four-arm counter telescope and measures the proton-proton elastic scattering events from a polyethylene block target. ${ }^{25)}$ However, in oraer to tune the polarized beam through the depolarizing resonances with minimum polarization loss, it is quite necessary to measure the beam polarization before and after each depolarizing resonance. For this purpose, the internal polarimeter is the best one to measure beam polarization during the acceleration cycle. This will generally analyze proton-proton elastic scattering from the hydrogen in polyethylene or nylon. ${ }^{4), 22), 26)}$

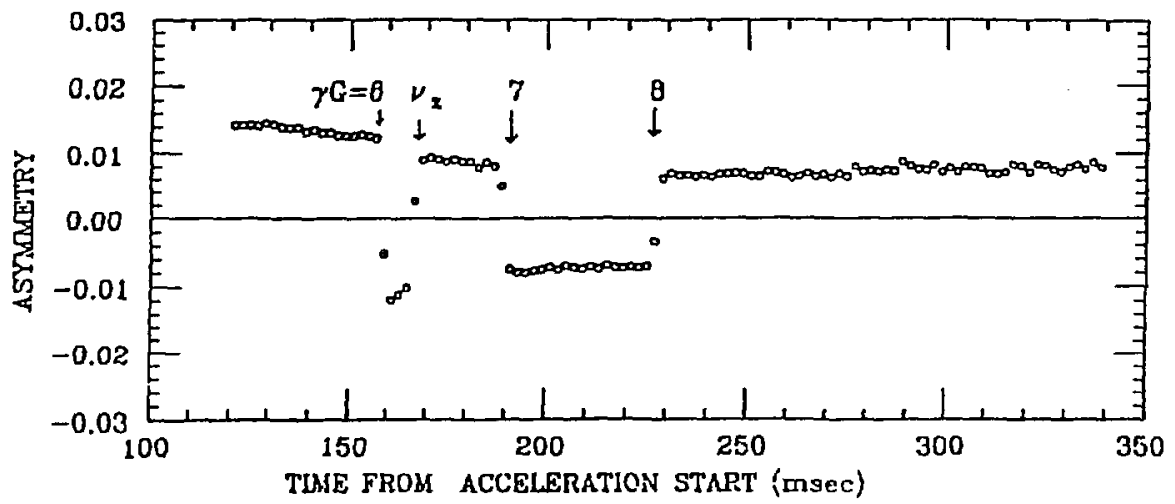

Fig.14. Continuous asymmetry measurment with a fixed angle back ward counter telescope during acceleration up to $3.5 \mathrm{GeV}$. 
Especially, the continuous asymmetry measurement during acceleration is quite useful to identify the depolarizing position. For the KEK PS, the beam intensity and target thickness are sufficiently adequate, and this method has been used successfully as shown in Fig 14.27).28) Furthermore this internal polarimeter was used for the experiment to measure the asymmetry in proton-proton elastic scattering during acceleration. ${ }^{6)}$ The use of the internal polarimeter at the AGS in an energy scan mode caused a significant polarization loss due to the emittance blow up by the multiple scattering in the target material, because the beam intensity is high and target is rather thick to use this method. A thick target is necessary since the Analyzing Power drops significantly with energy. However, even if the measurement period would be about $100 \mathrm{msec}$, this method may give much information on several resonances. ${ }^{27}$ )

\section{Conclusions}

It seems that the conventional techniques of polarized proton beam acceleration in the strong focusing synchrotron, such as the fast tune jump, COD harmonic correction and adiabatic spin flip, are well established up to a few tens GeV. This paper has reported on the phenomena which have been encountered in acceleration of the polarized proton beam especially at the AGS and KEK PS. It becomes clear that the several problems still remain, and more detailed work by both theoretical and technical approaches is necessary to obtain the larger polarization of the beam in the region of less than few tens of $\mathrm{GeV}$.

Finally the author would like to describe briefly the Siberian snake which is an attractive method to accelerate polarized protons to high energy. This was first proposed by Derbenev and Kondratenko in 1974. ${ }^{29)}$ Recently, the first experimental test of the Siberian snake 
concept has been performed at the Indiana University Cooler Ring by the Michigan-Indiana-Brookhaven team. ${ }^{30)}$ It overcame the imperfection resonance at $108 \mathrm{MeV}$ and the intrinsic resonance at $177 \mathrm{MeV}$. The Siberian snake may be feasible to accelerate polarized beam to high energy without tricky work such as fast tune jump and harmonic correction of COD, and it might be used at the AGS. At the KEK PS, it is difficult to situate the snake magnet, because there is no suitable long straight section in the ring. However, if the depolarizing resonances are not so strong a partial snake method ${ }^{31}$ ) (which rotates the spin by some small angle not $180^{\circ}$ ) might be feasible to maintain the beam polarization while passing through them. A feasibility study of a partial snake for the KEK PS is needed.

* S.Y.Lee proposed $3 \varepsilon$ to give $95 \%$ preservation of polarization. ${ }^{14)}$

** L.G.Ratner suggested that the value written in Ref.5, which is different in table 2, was misprinted.

\section{Acknowledgement}

The author wishes to express his gratitude to Dr.L.G.Ratner and Dr.L.A.Ahrens for providing the data of polarized proton beam acceleration at the AGS and for their helpful discussions. He is also grateful to Dr.S.Y.Lee, Dr.S.Hiramatsu, Dr.T.Toyama and Dr.J.A.Holt for their helpful discussions. This report has been prepared during his stay at Brookhaven National Laboratory. He would also like to thank Professor M.Kihara and many persons at KEK for giving him a chance 
and assistance to visit BNL and he also thanks many BNL staff members for their kind reception.

\section{References}

1) J.Arvieux, Proc. of the 8th Int. Symp. on High Energy Spin Physics, Minneapolis, 1988, AIP Conf. Proc. 187(1989)1191

2) L.A.Ahrens, Proc. of the 8th Int. Symp. on High Energy Spin Physics, Minneapolis, 1988, AIP Conf. Proc. 187(1989)1058

3) S.Hiramatsu et al., Proc. of the 8th Int. Symp. on High Energy Spin Physics, Minneapolis, 1988, AIP Conf. Proc. 187(1989)1077

4) F.Z.Khiari et al., Phys.Rev.D39(1989)45

5) C.Ohmori et al., Phys.Lett. B230(1989)27

6) H.Shimizu et al., Invited talk at the Sth French-Japanese Symposium on Nuclear Physics, Dogashima, Japan, Sep.26-30, 1989

7) M.Froissart and R.Stora, Nucl.Instrum. \& Methods 7(1960)297

8) K.M.Terwilliger et al., IEEE Trans. on Nucl. Sci..NS-28(1981)2031

9) H.Sato, Japanese Joumal of Applied Physics 27(1988)1022

10) H.Sato et al., Nucl.Instrum. \& Methods A272(1988)617

11) H.Sato et al., KEK 87-22

12) L.G.Ratner et al., IEEE Trans. on Nucl. Sci.,NS-32(1985)1656 and Proc. of the 6th Int. Symp. on High Energy Spin Physics, Marseille, 1984, p.c2-625

13) R.J.Nawrocky and R.F.Lambiase, IEEE Trans. on Nucl. Sci., NS-30(1983) 2772

14) S.Y.Lee, Proc. of the 8th Int. Symp. on High Energy Spin Physics, Minneapolis, 1988, AIP Conf. Proc. 187(1989)1 105 and also BNL-42365

15) E.D.Courant and H.S.Snyder, Annals of Physics 3(1958)1 
16) L.G. Ratner, BNL-41370, 3rd Conf.on the Intersection between Particle and Nuclear Physics, Rockport,Maine,May 1988

17) E.Groud et al., Proc. of the Sth Int. Symp. on High Energy Spin Physics, BNL, 1982, AIP Conf. Proc. 95(1982)407

18) S.Hiramatsu et al., Proc. of the 8th Int. Symp. on High Energy Spin Physics, Minneapolis, 1988, AIP Conf. Proc. 187(1989)1436

19) T.Toyama et al., KEK Preprint 89-112, Submirted to the 14th Int. Conf. on High Energy Accelerators, Tsukuba, Japan, 1989

20) K.M.Terwilliger et al., IEEE Trans. on Nucl. Sci.,NS-32(1985)2635

21) T.Aniel et al., Proc. of the 6th Int. Symp. on High Energy Spin

Physics, Marseille, 1984, p.c2-499

22) L.G. Ratner, IEEE Trans. on Nucl. Sci.,NS-3G(1983)2690

23) H.Sato et al., KEK Preprint 87-147

24) A.D.Krisch , Proc. of the 7th Int. Symp. on High Energy Spin Physics, Protvino, 1986, p.41

25) C.Ohmori et al., Nucl.Instrum. \& Methods in Phys.Res. A278(1989)705

26). H.Sato et al., IEEE Trans. on Nucl. Sci.,NS-32(1985) 1950

27) H.Sato et al., Proc.of the 1989 IEEE Particle Conference, Chicago, March 20-23, 1989, p.1758

28) H.Sato, AGS/AD/Tech.Note No.322,1989

29) Ya.S.Derbenev and A.M.Kondratenko, Part.Accel.8(1978)115

30) A.D.Krisch et al., Phys.Rev.Lett. 63(1989)1137

31) T.Roser, Proc. of the 8th Int. Symp. on High Energy Spin Physics, Minneapolis, 1988, AIP Conf. Proc. 187(1989)1442 